\title{
Aggressive Angiomyxoid Tumour - A Very Rare Pathologic Finding in the Urinary Bladder Co-existing with Pregnancy
}

\section{OBI-NJOKU OBINNA*, Coscione Alberto and Hiten Patel}

The Princess Alexandra Hospital, Hamstel Road Harlow, Essex, UK

*Corresponding author: OBI-NJOKU OBINNA, The Princess Alexandra Hospital, Hamstel Road Harlow, Essex, UK, Tel: +447947192090, E-mail: obinna.obi-njoku@pah.nhs.uk

\begin{abstract}
The bladder is an uncommon site for an aggressive Angiomyxoid tumour. We present a case of a 23-year-old primigravida who suffered no urinary symptoms and was referred due to an incidental bladder finding on ultrasound scan and went on to have a rigid cystoscopy and resection of lesion which was confirmed by histology to be an aggressive Angiomyxoid tumour. We include a review of most recent literature, and propose that, even in pregnancy, aggressive Angiomyxoid tumours can be appropriately managed by surgical excision and surveillance cystoscopy.
\end{abstract}

\section{Case Report}

A 23-year-old primigravida with no urinary symptoms was referred to our urology service following an

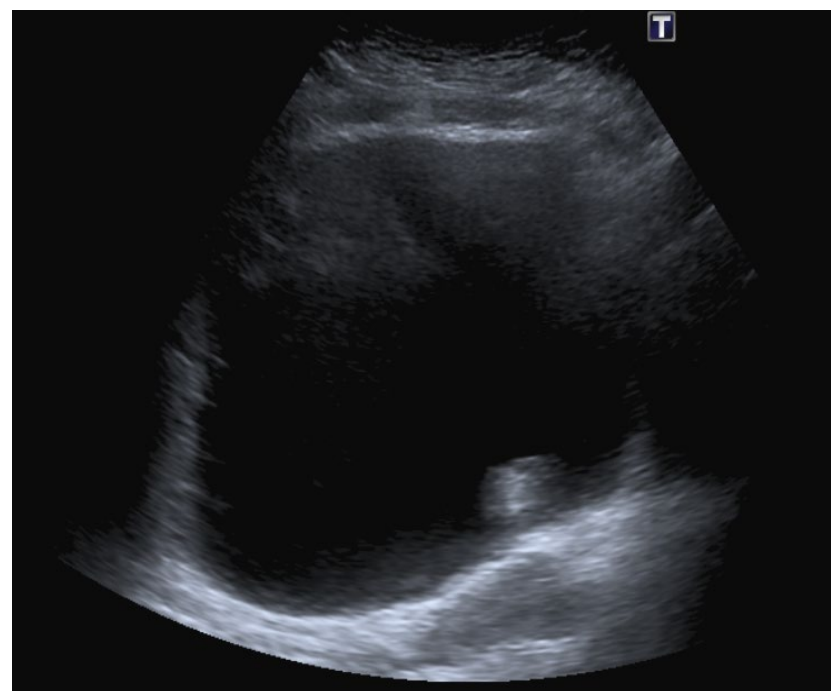

Figure 1a: Aggressive Angiomyxoid tumour in bladder, AP view as seen on USS scan.

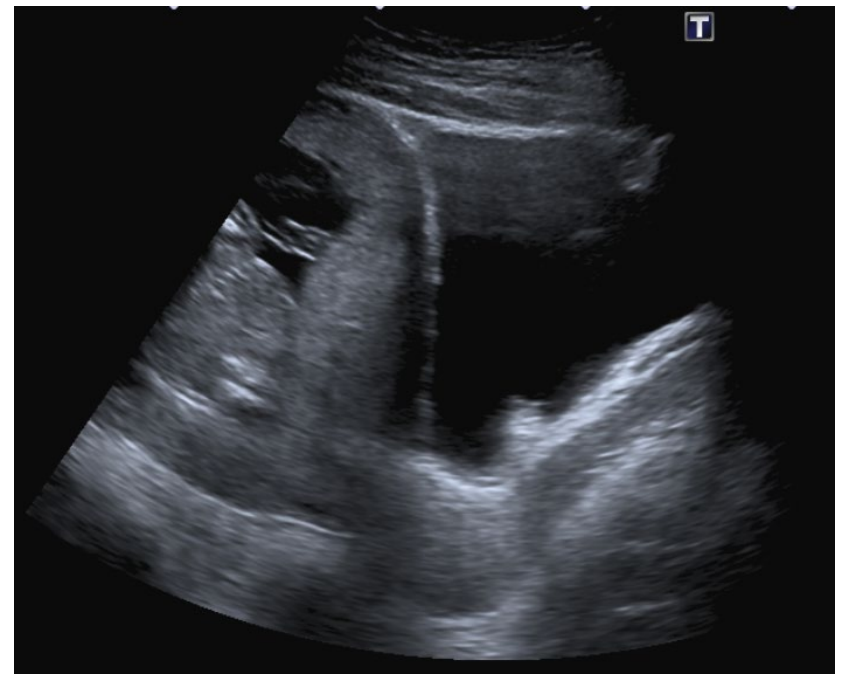

Figure 1b: Aggressive Angiomyxoid tumour in bladder, Lateral view as seen on USS scan.

incidental finding of a bladder lesion on ultrasound during a routine antenatal appointment at 12 weeks gestation. The lesion was described as measuring 12 $\times 15 \times 13 \mathrm{~mm}$ with no increased vascularity and present on the posterior bladder wall close to the left Vesico-Ureteric Junction (VUJ) as seen in Figure 1a and Figure 1b. She is a non-smoker with family history of bladder cancer but no previous urological history and is otherwise fit and well.

She was discussed in the uro-oncology Multidisciplinary Team meeting (MDT) and underwent an ultrasound scan of urinary tracts which showed presence of mucosal projection appearing as persistent filling defect in the bladder as seen in Figure $1 \mathrm{a}$ and Figure $1 \mathrm{~b}$ in 


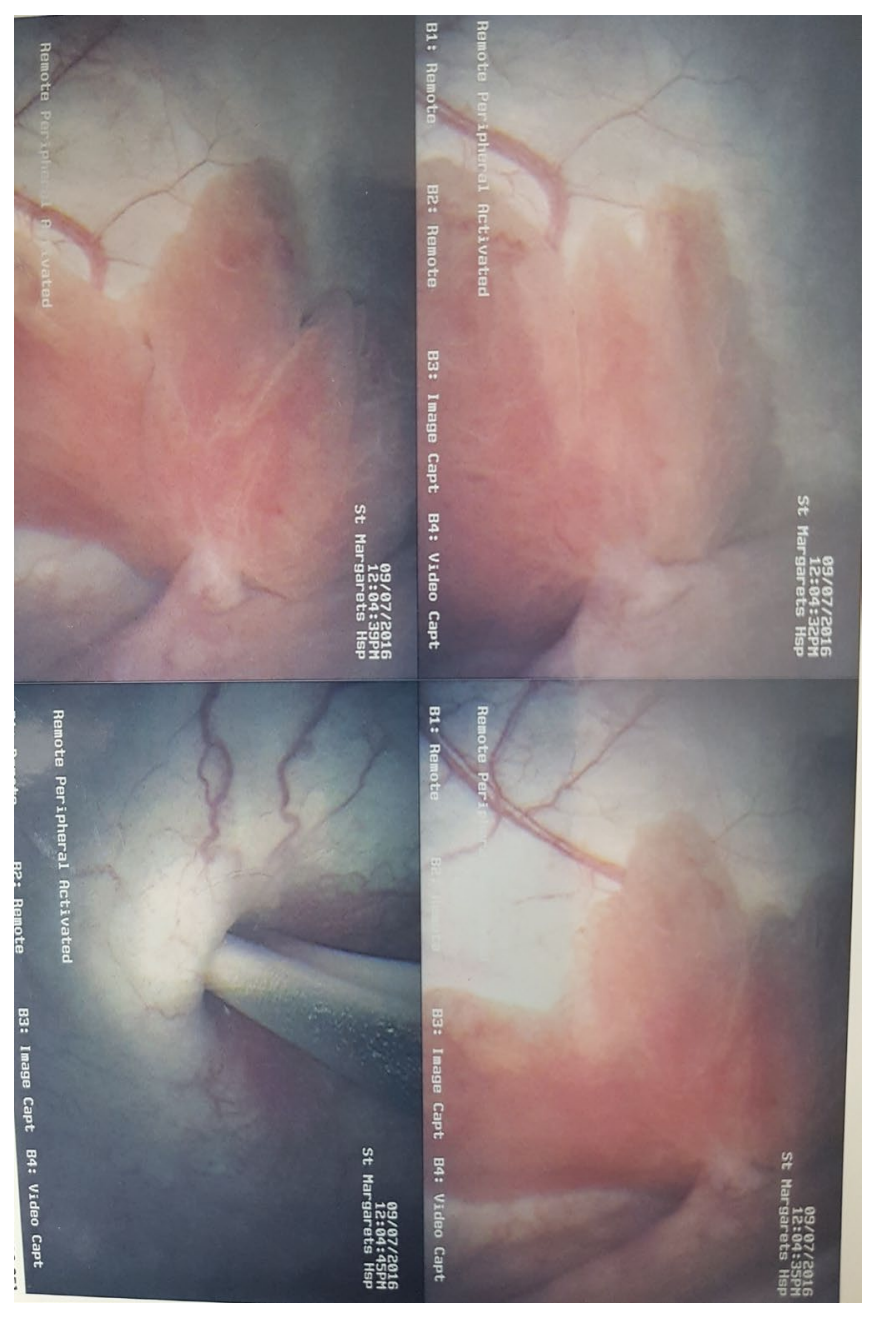

A

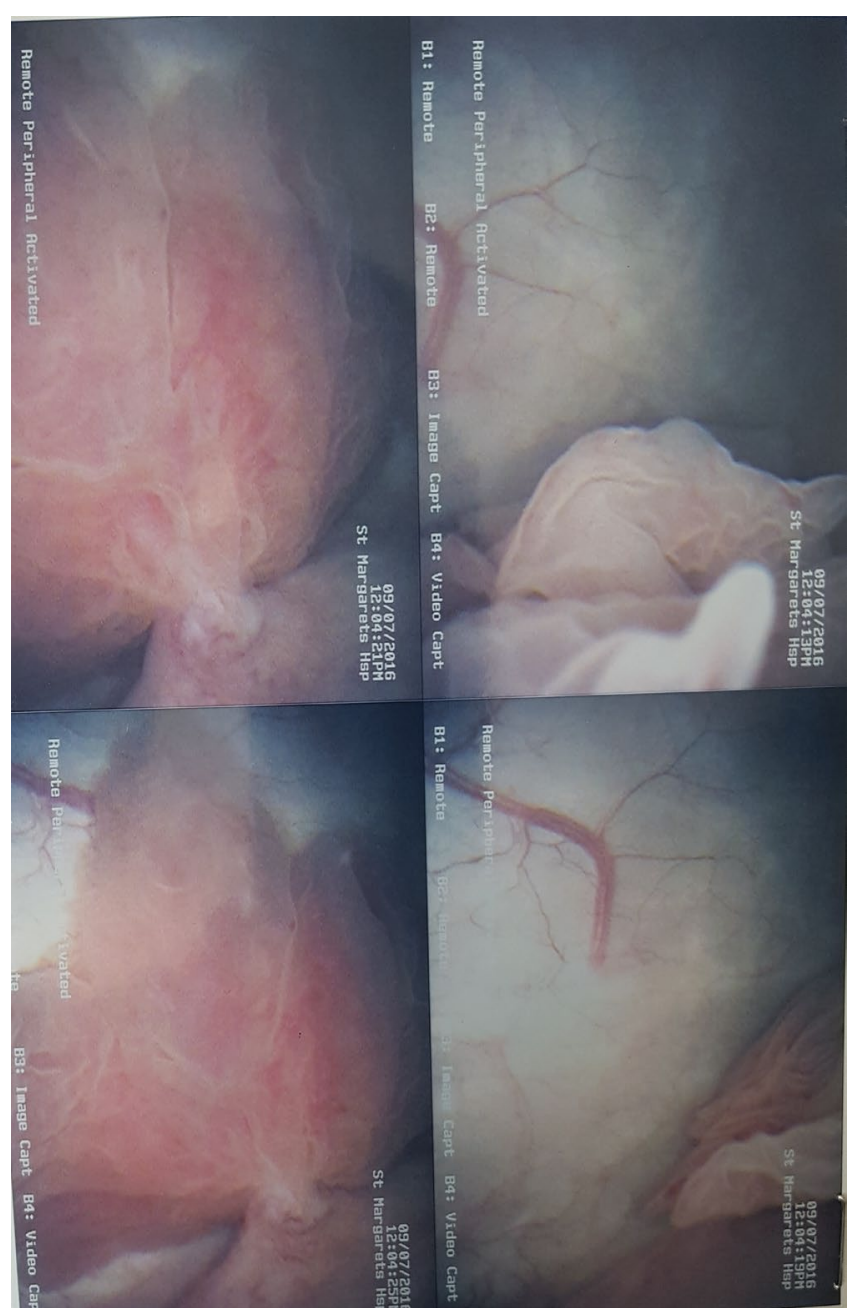

B

Figure 2: Aggressive Angiomyxoid tumour as seen in the bladder on flexible cystoscopy.

an otherwise normal urinary tract but nothing to differentiate it from a transitional cell carcinoma.

Flexible Cystoscopy described a solitary papillary growth with a thin stalk arising next to the left ureteric orifice, Figure $2 a$ and Figure $2 b$. Urine cytology was negative for malignant or atypical cells.

The presence of the rare bladder angiomyxoma in the gravid patient and the need to exclude any possibility of cancer posed a challenge and required a multidisciplinary approach involving the pathology, oncology, urology, gynaecology and sarcoma team collaboration.

Following discussion in our multidisciplinary team meeting and counselling of the patient, she was reviewed in the high-risk anaesthetic clinic and underwent a rigid cystoscopy and Transurethral Resection of Bladder Tumour (TURBT) under epidural anaesthesia. Findings revealed a $12 \times 15 \times 13 \mathrm{~mm}$ exophytic lesion behind left ureteric orifice as seen in Figure 3. Following the procedure, she was catheterised, and no complications were recorded.

Following resection of tumour, histology assessment including histomorphologic and immunohistochemi-

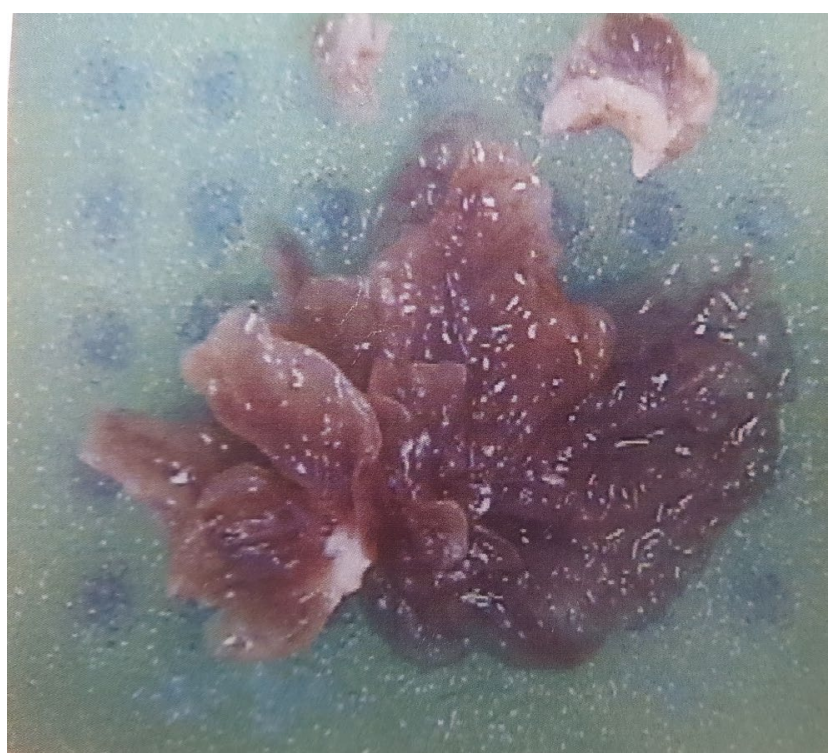

Figure 3: Macroscopic appearance of aggressive Angiomyxoid tumour post resection from bladder.

cal analysis demonstrated microscopic unremarkable urothelium with underlying stroma showing a tumour composed of numerous congested blood vessels of vari- 


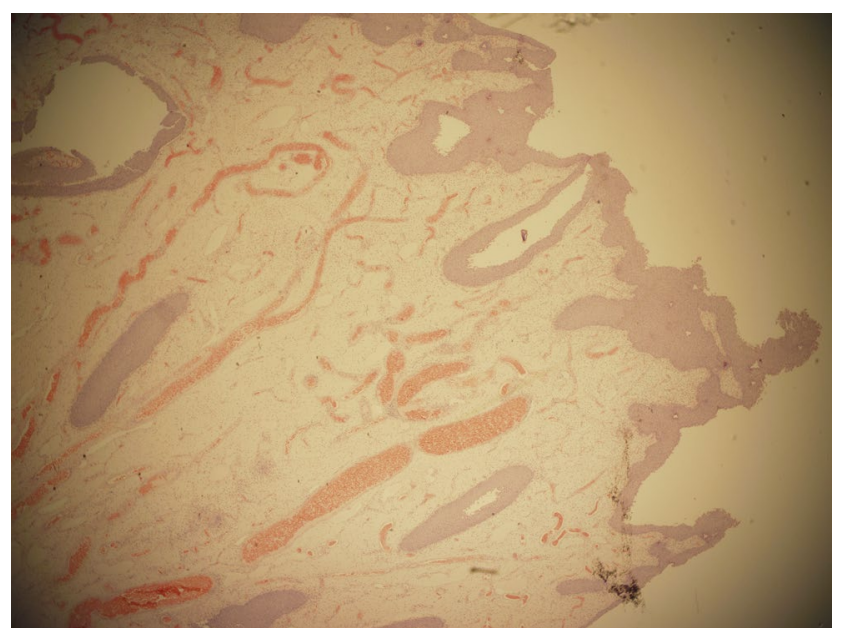

Figure 4a: Microscopic appearance of aggressive Angiomyxoid tumour under low power resolution.

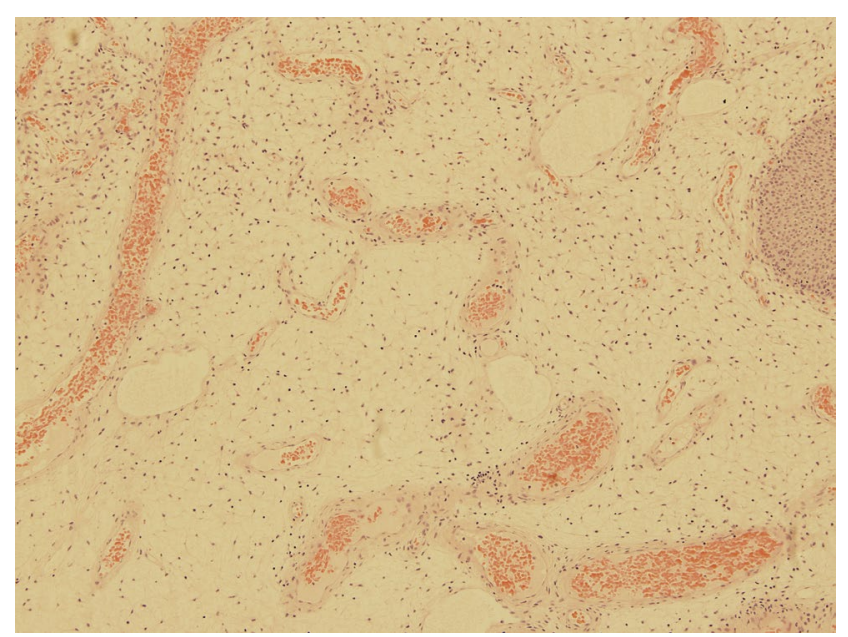

Figure 4c: Microscopic appearance of aggressive Angiomyxoid tumour under high power resolution.

able sizes and shapes with a hypocellular myxoid stroma containing spindle, oval and stellate shaped cells with no evidence of mitosis, nuclear pleomorphism, necrosis or haemorrhage seen; as seen in Figure 4a, Figure 4b, Figure 4c. Furthermore, the tumour cells showed positive immunostaining with vimentin; Figure $4 d$ and were uniformly negative with SMA, CD34, desmin, myosin, S100 and Ki67. In addition, ER and PGR showed widespread positivity while AE1/AE3, EMA and MUC4 were negative.

The overall morphological appearances together with the immunohistochemical staining profile were suggestive of an aggressive Angiomyxoid tumour ruling out possible differentials such as rhabdomyosarcoma, transitional cell carcinoma, tuberosclerosis, angiomyolipoma and liposarcoma.

In view of high probability of recurrence, a follow-up plan was recommended by MDT which involved a six-monthly flexible cystoscopy. In case of recurrence, trans-urethral resection and random bladder biopsies under anaesthesia with CT - urogram will be performed. However, she went on to have an MRI as she was gravid

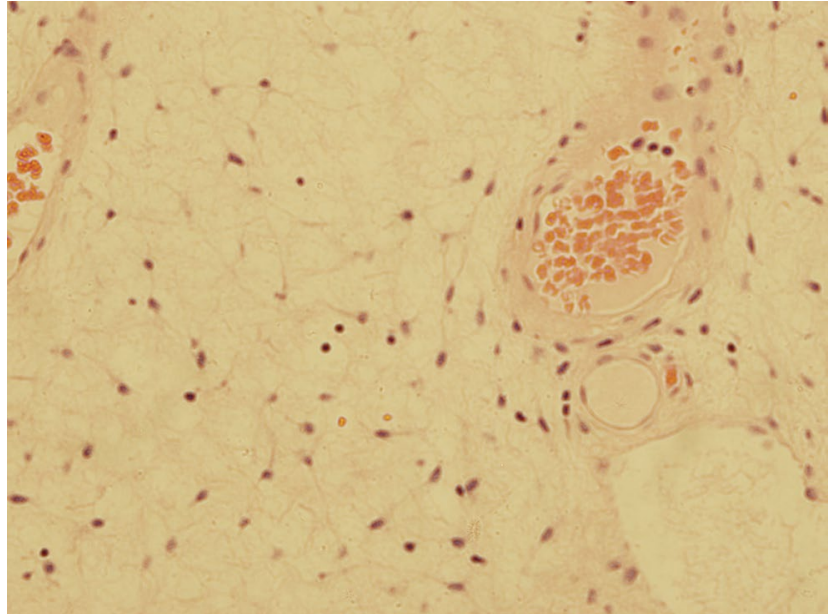

Figure 4b: Microscopic appearance of aggressive Angiomyxoid tumour under moderate power resolution.

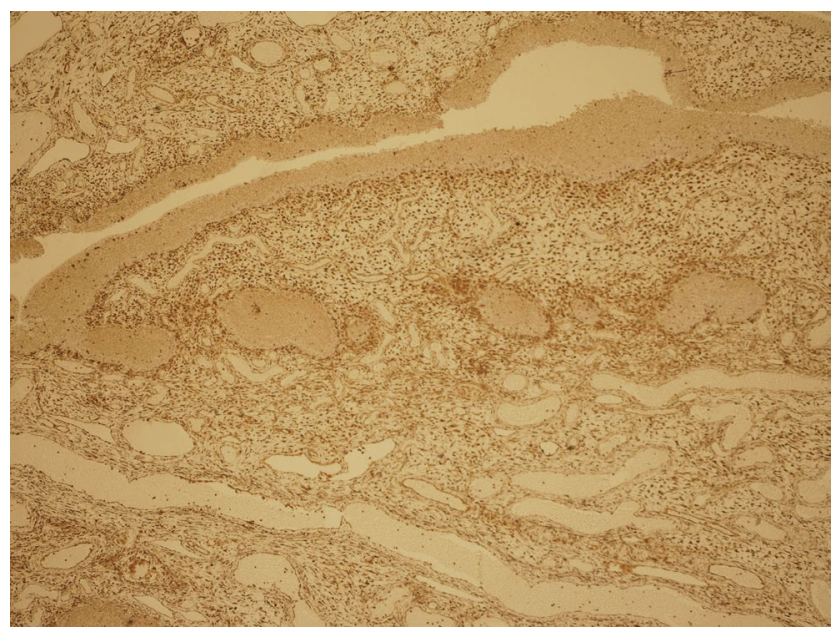

Figure 4d: Microscopic appearance of aggressive Angiomyxoid with vimentin immunohistochemistry.

which revealed normal urinary tract and flexible cystoscopy done 6 months later revealed no recurrence; Figure 5 and Figure 6 respectively.

\section{Discussion}

Aggressive Angiomyxoma is a rare connective tissue tumour involving myxoid cells and blood vessels. After a review of world literature, it is notable to say that this is the first ever report of aggressive angiomyxoma found in the bladder during pregnancy and the fifth report of this benign tumour originating primarily in the bladder making the bladder a very rare site for aggressive angiomyxoma and even extremely rare in pregnancy [1-4]. In addition, this is the fourteenth report of Aggressive Angiomyxoma coexisting in pregnancy, but the bladder has never been a preferred site until now [5-20].

They are rare mesenchymal tumours which tend to affect fertile females in the $4^{\text {th }}$ to $5^{\text {th }}$ decade of life [7] but can also affect younger females as found in this case. Although technically benign tumours involving mainly the pelvis, vulva, perineum, vagina and urinary bladder in adult women of reproductive age, the prefix aggressive 


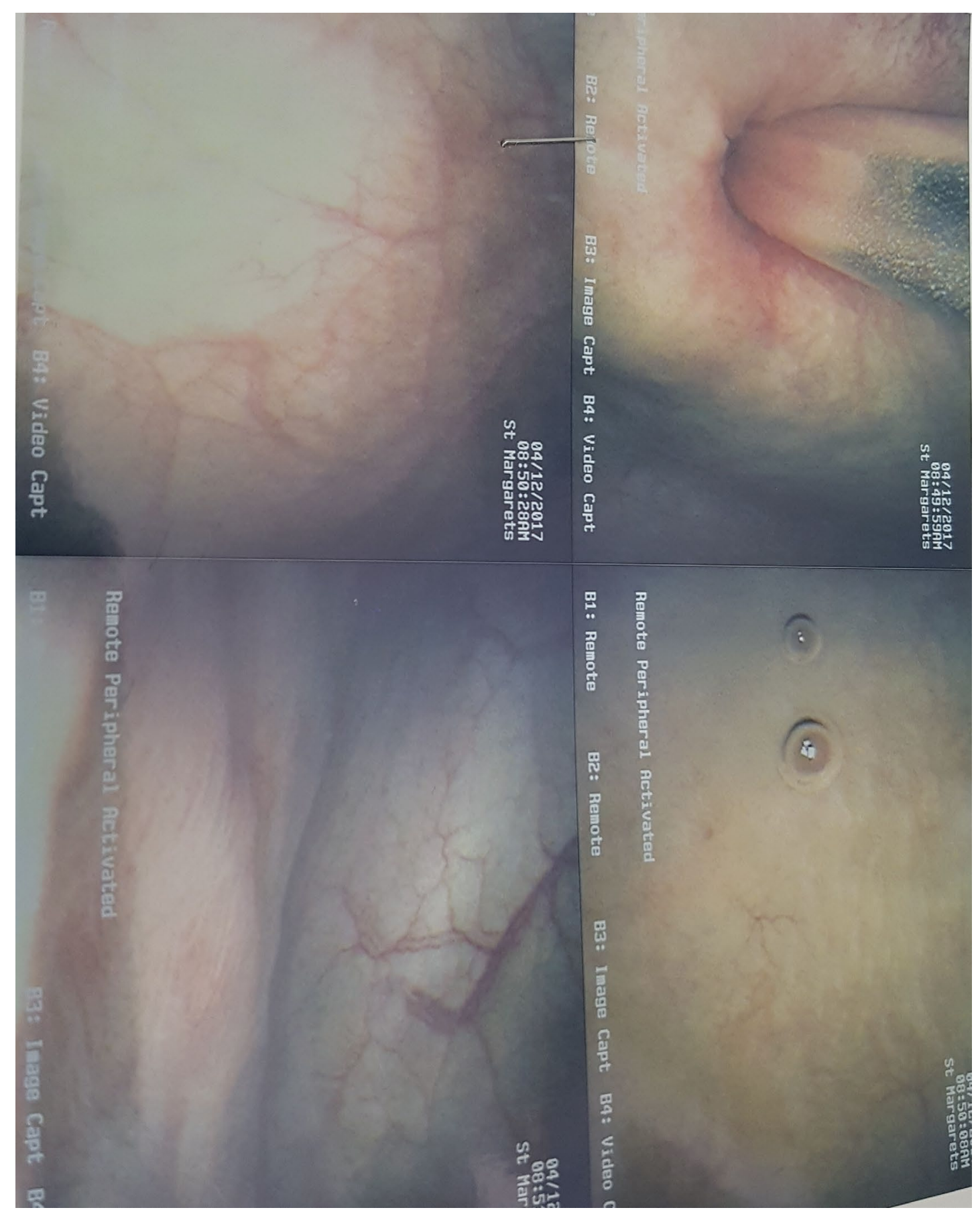

Figure 5: Surveillance flexible cystoscopic 6 months post resection - no recurrence) post resection 1 - flexi.

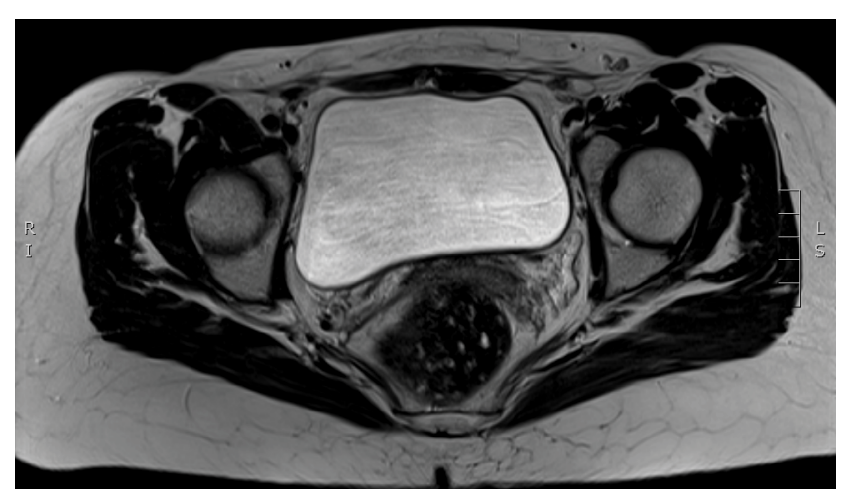

Figure 6: Post resection MRI showing no bladder recurrence.

was added to highlight their tendency to infiltrate surrounding tissues and recur after treatment [21]. Recur- rence rates range from $25-47 \%$, usually within 5 years and cases of metastasis have been reported; hence radiological follow up is advocated [6].

On the other hand, the other type of angiomyxoma has a preferred location different from aggressive angiomyxoma and grows on the external surface of the body such as external genitalia, head and neck regions and named superficial angiomyxoma. Aggressive Angiomyxoma has a female: male sex distribution of $6.6: 1$ presenting as painless perineal or vaginal masses with few other associated symptoms [7]. Haematuria has also been noted as a presenting feature of this rare disease [1]. It is still broadly asymptomatic and presents with non-specific symptoms, thus a high index of suspicion is required in making accurate diagnosis and excluding 
likely differentials. Though commonly found in fertile females, it can also be found in males as once reported in a case where a patient presented with acute retention of urine and further investigation revealed presence of aggressive angiomyxoma originating from the prostate and fanning into the bladder neck [8].

It is likely to grow during pregnancy and do respond to hormonal manipulation due to the presence of estrogen and progesterone receptors [21]. The hormonal dependency of this tumour is further supported in another case report where it showed an increased growth of the tumour during pregnancy and often misdiagnosed resulting in the delay of the treatment [5]. This is evident in this case as it was an incidental finding in early pregnancy. Perhaps it was present before conception.

The mainstay of treatment is wide local resection. However, recurrence is still high and independent of resection margin, as confirmed in a study where seventy-one percent of recurrence occurred within the first 3 years of resection and patients with clear resection margins had similar chances of remaining disease-free compared to those having tumor-involved resection margins [6]. Hence the need for surveillance.

Overall, the findings been made suggests the benign nature of aggressive angiomyxoma, its high recurrent rate and a rare probability of malignant change which is nonetheless not entirely impossible. As a result, we propose that even if asymptomatic as well as in pregnan$c y$, the management of aggressive angiomyxoma in the bladder should be surgical excision and diagnosis confirmed with both histomorphologic and immunohistochemical analysis and follow up with active surveillance using cystoscopy and imaging.

\section{MRI and Sonographic Appearance of Aggressive} Angiomyxoma - No Specific or Distinguishing Pattern Seen

In this case, the patient had an obstetric ultrasound scan which revealed a $12 \times 15 \times 13 \mathrm{~mm}$ soft tissue lesion on the posterior bladder wall close to the left VUJ and no increased vascularity detected on Color Doppler. In addition, a repeat Ultrasound scan done revealed the presence of mucosal projection appearing as persistent filling defect in the bladder as seen in Figure 1a and Figure $1 \mathrm{~b}$ but nothing to differentiate it from a transitional cell carcinoma. Unfortunately, MRI was only done post resection which however revealed a normal urothelium.

A review of literature reported a case on the sonographic appearance of a histologically proven aggressive angiomyxoma of the bladder [3]. The findings revealed a solid polypoid mass $2 \mathrm{~cm}$ in diameter with sharply demarcated borders, a heterogeneous echotexture and a slightly hyperechoic rim.

In another report of bladder angiomyxoma, MRI revealed an $85 \times 40 \mathrm{~mm}$ mass arising from the bladder neck which appeared hypo intense on T1-sequence and had a swirled, heterogeneous appearance on contrast enhanced T2-sequence [1].

From the above literatures, there seems to be no specific features on imaging suggestive of aggressive angiomyxoma and as well no distinguishing features suggestive of being benign or malignant in nature. As a result, we recommend that any lesion in the bladder detected on USS or MRI which are both safe in pregnancy, should have further evaluation and eventual resection even in pregnancy.

Consideration of biopsy before tumor resection will not alter management protocol even in pregnancy as suggested in a report where a review of literature failed to find any evidence that pregnancy has deleterious effects on the incidence or clinical course of malignancy compared with that in nonpregnant women of reproductive age, except in the case of malignant melanoma [22]. By extension, this suggests that pregnancy should not alter management protocol in any way be it benign or malignant and as such every tumor should be treated in the same way.

In our case and in most other cases where bladder tumor was found to co-exist with pregnancy, Transurethral resection of the tumor was the primary treatment regardless of the trimester and treatment was never delayed due to pregnancy. A review of different literature has shown the safety of Transurethral resection of bladder tumor under regional or general anaesthesia at any time during pregnancy [22-27].

In this case, a bladder tumor was incidentally discovered on obstetric ultrasound and described as a $12 \times$ $15 \times 13 \mathrm{~mm}$ soft tissue lesion on the posterior bladder wall close to the left VUJ with no increased vascularity detected on Color Doppler. Meanwhile, a repeat Ultrasound scan done revealed the presence of mucosal projection from the bladder as seen in Figure 1a and Figure $1 \mathrm{~b}$ but nothing to differentiate it from a transitional cell carcinoma. We went on to review this patient's case in the MDT and the opinion was to go ahead with a flexible cystoscopy in order to confirm sonographic findings. The fact that bladder carcinoma is not uncommon in this age group, [22] and ultrasound scan alone cannot differentiate a benign from malignant lesion strengthened the decision made on the day.

The cystoscopy finding revealed a solitary papillary growth with a thin stalk arising next to the left ureteric orifice as seen in Figure 3a and Figure 3b. Possibly a biopsy of the tumour could have been done at this point as was the case in a certain report, [23] to confirm or rule out presence of malignancy in order to consider whether a resection was necessary at this stage in pregnancy in order to defer treatment till post-partum. However, bearing in mind the local policy of TURBT for superficial bladder lesion and evidence from various lit- 
eratures review suggesting safety of this procedure in pregnancy she went on to have resection of the tumour and eventually had a safe delivery afterwards.

In addition, the safety of TURBT at any time in pregnancy can be shown by our case and more so in a case where the patient had Transurethral resections under general anesthesia on three different occasions for recurrent bladder tumour and yet had an uneventful delivery of a healthy and thriving baby thereafter [22]. A review to evaluate tumour presentation, characteristics and maternal-foetal outcomes of pregnancies complicated by nonbilharzial bladder carcinoma revealed that treatment was typically by transurethral resection and pregnancy is not a contraindication to treating most forms of bladder cancer [25].

In conclusion, TURBT is safe at any time during pregnancy and there is no added advantage having a biopsy before actual resection of the tumour. Finally, we still propose Transurethral resection of bladder tumour under regional or general anaesthesia as the primary treatment of any bladder tumour during pregnancy.

\section{References}

1. Davari M, Lamb BW, Chowdhury S, Jameson C, Kelly JD, et al. (2015) Aggressive Angiomyxoma of the Bladder Neck Requiring Local Excision and Mitrofanoff Formation. Case Rep Urol 2015: 819243.

2. May F, Luther A, Mohr W, Bachor R, Hautmann RE (2000) Recurrent aggressive angiomyxoma of the urinary bladder. Case report and review of the literature. Urol Int 65: 57-59.

3. Cesarani F, Garretti L, Denegri F, Valente G, Rossetti SR (1999) Sonographic appearance of aggressive angiomyxoma of the bladder. J Clin Ultrasound 27: 399-401.

4. López López C, Romero Maroto J, Quílez Fenoll JM, Mayol Belda MJ, Boldó Díaz A (1993) New location of aggressive angiomyxoma: the bladder. Actas Urol Esp 17: 611-613.

5. Orfanelli T, Kim CS, Vitez SF, Van Gurp J, Misra N (2016) A Case Report of Aggressive Angiomyxoma in Pregnancy: Do Hormones Play a Role? Case Rep Obstet Gynecol 2016: 6810368 .

6. Chan YM, Hon E, Ngai SW, Ng TY, Wong LC (2000) Aggressive angiomyxoma in females: is radical resection the only option? Acta Obstet Gynecol Scand 79: 216-220.

7. Sutton BJ, Laudadio J (2012) Aggressive angiomyxoma Arch Pathol Lab Med 136: 217-221.

8. Sawada $\mathrm{Y}$, Ito $\mathrm{F}$, Nakazawa $\mathrm{H}$, Tsushima $\mathrm{N}$, Tomoe $\mathrm{H}$, et al. (2010) A rare benign genitourinary tumor in a Japanese male: urinary retention owing to aggressive angiomyxoma of the prostate. Rare Tumors 2: e15.

9. Zangmo R, Kumar S, Singh N, Meena J (2016) Aggressive Angiomyxoma of Vulva in Pregnancy: A Case Report. J Obstet Gynaecol India 66: 610-612.

10. Sampaio J, Sarmento-Gonçalves I, Ramada D, Amaro T, Tiago-Silva P (2016) Aggressive Angiomyxoma in Pregnancy: A Rare Condition, a Common Misdiagnosis. Case Rep Obstet Gynecol 2016: 8539704.

11. Sinha V, Dave KS, Bhansali RP, Arora RS (2014) Aggressive angiomyxoma of vulva which grew with pregnancy and attained a huge size rarely seen in literature. J Obstet Gy- naecol India 64: 90-91.

12. Goyal P, Agrawal D, Sehgal S, Ghosh S, Kumar A, et al. (2014) Aggressive angiomyxoma in pregnancy. Rare Tumors 6: 5362.

13. Ashraf T, Haroon S (2014) Aggressive angiomyxoma in pregnancy. J Coll Physicians Surg Pak 24: S24-S26.

14. Aye C, Jefferis H, Chung DY, Manek S, Kehoe S (2009) A case of multi-modal managed vulval aggressive angiomyxoma diagnosed before conception and monitored during pregnancy. Gynecol Oncol 115: 170-171.

15. Mandal S, Dhingra K, Roy S, Khurana N (2008) Aggressive angiomyxoma of the vulva presenting as a pedunculated swelling. Indian J Pathol Microbiol 51: 259-260.

16. Bagga R, Keepanasseril A, Suri V, Nijhawan R (2007) Aggressive angiomyxoma of the vulva in pregnancy: a case report and review of management options. MedGenMed 9: 16.

17. Ribaldone R, Piantanida P, Surico D, Boldorini R, Colombo $\mathrm{N}$, et al. (2004) Aggressive angiomyxoma of the vulva. Gynecol Oncol 95: 724-728.

18. Wolf CA, Kurzeja R, Fietze E, Buscher U (2003) Aggressive angiomyxoma of the female perineum in pregnancy. Acta Obstet Gynecol Scand 82: 484-485.

19. Smirniotis V, Kondi-Pafiti A, Theodoraki K, Kostopanagiotou G, Liapis A, et al. (1997) Aggressive angiomyxoma of the pelvis: a clinicopathologic study of a case. Clin Exp Obstet Gynecol 24: 209-211.

20. Htwe M, Deppisch LM, Saint-Julien JS (1995) Hormone-dependent, aggressive angiomyxoma of the vulva. Obstet Gynecol 86: 697-699.

21. Kura MM, Jindal SR, Khemani UN (2012) Aggressive angiomyxoma of the vulva: An uncommon entity. Indian Dermatol Online J 3: 128-130.

22. Lakmichi MA, Zehraoui R, Dahami Z, Moudouni SM, Bassir A, et al. (2012) Bladder cancer in the second trimester of pregnancy: tough decisions. A case report with review of the literature. Ther Adv Urol 4: 139-142.

23. Yeaton-Massey A, Brookfield KF, Aziz N, Mrazek-Pugh B, Chueh J (2013) Maternal bladder cancer diagnosed at routine first-trimester obstetric ultrasound examination. Obstet Gynecol 122: 464-467.

24. Gupta NP, Dorairajan LN (1997) Transurethral resection of a bladder tumor in pregnancy: a report of 2 cases. Int Urogynecol J Pelvic Floor Dysfunct 8: 230-232.

25. Wax JR, Pinette MG, Blackstone J, Cartin A, McCrann DJ (2002) Nonbilharzial bladder carcinoma complicating pregnancy: review of the literature. Obstet Gynecol Surv 57: 236-244.

26. Spahn M, Bader P, Westermann D, Echtle D, Frohneberg D (2005) Bladder carcinoma during pregnancy. Urol Int 74: 153-159.

27. Shrotri KN, Ross GC (2008) Bladder carcinoma presenting during twin pregnancy. J Obstet Gynaecol 28: 750-751. 